\title{
Understanding patients' perspective of statin therapy: can we design a better approach to the management of dyslipidaemia? A literature review
}

\author{
Ying Jie $\underline{C h e}^{1}$, MBBS, Hian Hui Vincent ${\underline{C h a n^{2}}}^{2}$, MBBS, MMed, Ngiap Chuan $\underline{\operatorname{Tan}}^{2,3}$, MMed, FCFPS
}

INTRODUCTION Dyslipidaemia leads to atherosclerosis and is a major risk factor for cardiovascular diseases. In clinical trials, 3-hydroxy-3-methylglutaryl-coenzyme A (HMG-CoA) reductase inhibitors, or statins, have been shown to effectively reduce dyslipidaemia. Despite the availability and accessibility of statins, myocardial infarctions and cerebrovascular accidents remain among the top causes of mortality in developed countries, including Singapore. This enigma could be attributed to suboptimal adherence to statin therapy. The present literature review aimed to evaluate patients' perceptions of statin therapy.

METHODS We searched PubMed and other databases for articles published in English from October 1991 to May 2012 containing keywords such as 'patient', 'views', 'perceptions', 'adherence', 'statin' and 'dyslipidaemia'. Of the 122 eligible studies retrieved, 58 were reviewed. The findings were categorised and framed in accordance with the Health Belief Model. RESULTS Patients with dyslipidaemia appeared to underestimate their susceptibility to dyslipidaemia-related complications, partly due to their demographic profiles. Failure to appreciate the severity of potential complications was a major hindrance toward adherence to statin therapy. Other factors that affected a patient's adherence included lack of perceived benefits, perceived side effects, the cost of statins, poor physician-patient relationship, and overestimation of the effectiveness of diet control as a treatment modality.

CONCLUSION Existing evidence suggests that the cause of poor adherence to statin therapy is multifactorial. The use of the Health Belief Model to present the results of our literature review provides a systematic framework that could be used to design a patient-centric approach for enhancing adherence to statin therapy.

Keywords: adherence, dyslipidaemia, patient, perceptions, statin

\section{INTRODUCTION}

Dyslipidaemia is a critical predisposing factor for the development of cardiovascular diseases. Managing dyslipidaemia, especially via the lowering of low-density lipoprotein ( $L D L)$, is pivotal to reducing cardiovascular complications. In a meta-analysis by Baigent et al involving more than 90,000 participants, LDL-lowering interventions were proven to be effective in significantly decreasing the risk of coronary artery disease and other vascular thrombotic diseases. ${ }^{(1}$

The advent of 3-hydroxy-3-methylglutaryl-coenzyme A (HMG-CoA) reductase inhibitors, or statins, has been a major medical breakthrough in the efforts to reduce LDL in patients with dyslipidaemia. A recent meta-analysis of 27 randomised trials conducted by the Cholesterol Treatment Trialists' Collaborators concluded that statins reduced the risk of major vascular events by $20 \% .^{(2)}$ However, although statins are readily available, control of dyslipidaemia remains suboptimal, with resultant myocardial infarction and strokes ranking among the top causes of mortality in many developed countries, including Singapore. Bates et al attributed this to the complex interactions among patient, physician and system factors, which affect the overall management of dyslipidaemia. ${ }^{(3)}$ Ho et al reported that, among a cohort of patients with coronary heart diseases from the Singapore National Cardiac Registry, 70\% did not achieve the serum LDL target of $<100 \mathrm{mg} / \mathrm{dL}$ (or $2.6 \mathrm{mmol} / \mathrm{L}$ ) and $94 \%$ of very high-risk patients did not achieve the stringent serum $\mathrm{LDL}$ target of $<70 \mathrm{mg} / \mathrm{dL}$ (or $1.8 \mathrm{mmol} / \mathrm{L}$ ). ${ }^{(4)}$ This phenomenon is paradoxical, as statins are affordable and readily available to patients via various healthcare institutions.

The lack of adherence to statin therapy is likely one of the factors contributing to this enigma. The optimal benefits of statins can only be reaped when patients take the medications. Failure to adhere to statin therapy exacts a toll on not only the patients, but also their families and communities, as the resultant higher rates of dyslipidaemia-related complications lead to poor quality of life, increased mortality, ${ }^{(5)}$ and an overall increase in healthcare costs. ${ }^{(6)}$ Adherence to statin therapy appears to be a major challenge worldwide. A study by Chaudhry and McDermott showed that the rate of adherence to statin therapy was only $50 \%$ at six months, and further declined at one year. ${ }^{(7)} \mathrm{A}$ similar finding was reproduced in a long-term follow-up of the West of Scotland Coronary Prevention Study, in which less than $40 \%$ of the patients adhered to statin therapy at five years after the trial. ${ }^{(8)}$ Benner et $\mathrm{a}^{(9)}$ further corroborated the issue of statin nonadherence by showing that only $25 \%$ of the participants in their study were adherent to statin therapy five years after it was initiated.

We conducted a literature review to determine patients' perceptions of statins, as well as the impact these perceptions had on statin use and adherence. A better understanding of patients' perceptions of statins is a prerequisite to designing a better

${ }^{1}$ Department of General Medicine, Tan Tock Seng Hospital, ${ }^{2}$ SingHealth Polyclinics - Pasir Ris, ${ }^{3}$ Duke-NUS Graduate Medical School, Singapore Correspondence: Dr Chee Ying Jie, House Officer, Department of General Medicine, Tan Tock Seng Hospital, 11 Jalan Tan Tock Seng, Singapore 308433 cheeyingjie.chee@mohh.com.sg. 
patient-centric approach that aims to improve the management of dysplipidaemia.

\section{METHODS}

A literature search for articles published from October 1991 to May 2012 was conducted on PubMed, Medscape, the Cochrane Database and the Western Pacific Region Index Medicus. The search terms included 'patient', 'views', 'perceptions', 'susceptibility', 'severity', 'barriers', 'adherence', 'compliance', 'HMG Co-A reductase inhibitor', 'statin', 'simvastatin', 'atorvastatin', 'lovastatin', 'interventions', 'costs', 'communication', 'relationship', 'diet', 'food', 'management', 'treatment', 'dyslipidaemia', 'hyperlipidaemia', and 'hypercholesterolaemia'. Additional studies were also identified using a manual reference search for included citations.

Article titles and abstracts were first screened to exclude studies that did not report factors affecting adherence or interventions that target to improve adherence. Non-English publications were excluded. A second screen was conducted to ensure that the content of the articles included factors relating to the use of medications in dyslipidaemia management, especially the use of statins. In the case of original research articles that identified factors influencing patients' perceptions on the use of statins, information on the methodology, target population and study outcomes were extracted. In the case of intervention-based articles, randomised controlled trials, non-randomised trials and meta-analyses were included. The types of interventions included in this literature review are patient education, medication reminders, medication cost management and enhancement of physician-patient communication.

\section{RESULTS}

A total of 122 studies were identified. Of these 122 studies, 58 were reviewed. We categorised the results of our literature review according to the key components of the Health Belief Model (HBM), ${ }^{(10)}$ which hypothesises that patients' health-related decisions are based on the following factors: (a) perceived susceptibility to a serious health problem; (b) perceived severity of the illness; (c) perceived benefits of the treatment in reducing susceptibility; and (d) perceived barriers restricting patients' use of the treatment. The HBM was used, as it provides a framework for easy reference, facilitating the elucidation of a patient-centric approach that incorporates factors that influence patients' views on the use of statins. These factors include the patients' demographic characteristics, perceived severity of and susceptibility to dyslipidaemia-related complications, and perceived benefits of and barriers to the use of statins. Fig. 1 summarises the results of the present literature review with regard to the aforementioned factors.

The practice of preventive behaviour is closely associated with patient demographics. Studies have suggested several patient demographic characteristics that contribute to medication adherence. A study by Wong et al, ${ }^{(11)}$ which adopted a computerised clinical database of 11,000 adult patients diagnosed with dyslipidaemia in Hong Kong, showed that the factors that contribute to optimal medication adherence included older age, the presence of a higher number of comorbidities, and regular follow-up with family medicine specialists. A systematic review of the predictors of nonadherence to statin therapy by Mann et $\mathrm{al}^{(12)}$ showed that female patients are less compliant with medications. This finding is supported by Chan et al's retrospective cohort study involving more than 14,000 patients. ${ }^{(13)}$ Both Mann et al and Chan et al also concluded that a higher median income was a significant predictor of adherence. ${ }^{(12,13)}$ In a review by Bates et al ${ }^{(3)}$ poor literacy was associated with lower medication compliance.

Perceived susceptibility to disease is one of the most influential components of the HBM that determines a patient's behaviour. ${ }^{(10)}$ However, as dyslipidaemia is a 'silent' disease, patients may not be conscious of their risk of adverse health outcomes. ${ }^{(14)}$ In fact, the asymptomatic nature of dyslipidaemia has been described by patients in focus group discussions as 'benign'. ${ }^{(14)}$ This observation is consistent with the findings of a series of focus group studies conducted by Goldman et al, which reported that some patients appeared nonchalant about the implications of the results of their lipid profiles. ${ }^{(15)}$ In fact, in a qualitative study reported by Fung et $\mathrm{al}^{\left({ }^{(16)}\right.}$ some participants even questioned their personal need for statins since there was an absence of symptoms related to hypercholesterolaemia. In short, patients' low perceived susceptibility to dyslipidaemia-related complications may lead to a low perceived need for lipid-lowering medications and poor medication adherence. Patient's perceived need for medication has been shown to be one of the best predictors of adherence. ${ }^{(17)}$ Mann et al found that a low perceived risk for acute myocardial infarction was a strong predictor of poor adherence to statins. ${ }^{(18)}$

Perceived severity of disease is influenced by personal views regarding the difficulties a health condition will inflict upon the sufferer. ${ }^{(19)}$ The impact of perceived severity on adherence to statin therapy remains inconclusive. In a meta-analysis by DiMatteo et $\mathrm{al}^{\left({ }^{(20)}\right.}$ perceived disease severity was found to be positively correlated with medication adherence. This finding is supported by Bates et al, ${ }^{(3)}$ who concluded in a review that previous cardiovascular events promote adherence to lipidlowering therapy. Yan et $\mathrm{al}^{(21)}$ assessed the illness perception of 193 Chinese patients after acute myocardial infarction and showed that patients who perceived myocardial infarction as a severe illness that could be controlled by medications were more likely to be adherent to lipid-lowering therapy. However, some studies also show that adherence to medication may not necessarily be improved in patients who had suffered from dyslipidaemia-related complications. For example, in a study by Choudhry et al ${ }^{(22)}$ involving over 33,000 patients, only $43.1 \%$ of the patients refilled their statin prescriptions within 90 days of hospital discharge after their first episode of acute myocardial infarction. Shah et $\mathrm{al}^{(23)}$ found that the three-year medication continuation rate for statins among 292 patients with a history of acute myocardial infarction was only $44 \%$. Nonadherence to statin therapy was also evident in patients who had suffered a stroke, with the statin discontinuation rates reaching 39\% one year after the initiation of therapy. ${ }^{(24)}$ However, it is worthwhile to note that these studies did not evaluate adherence to statin 


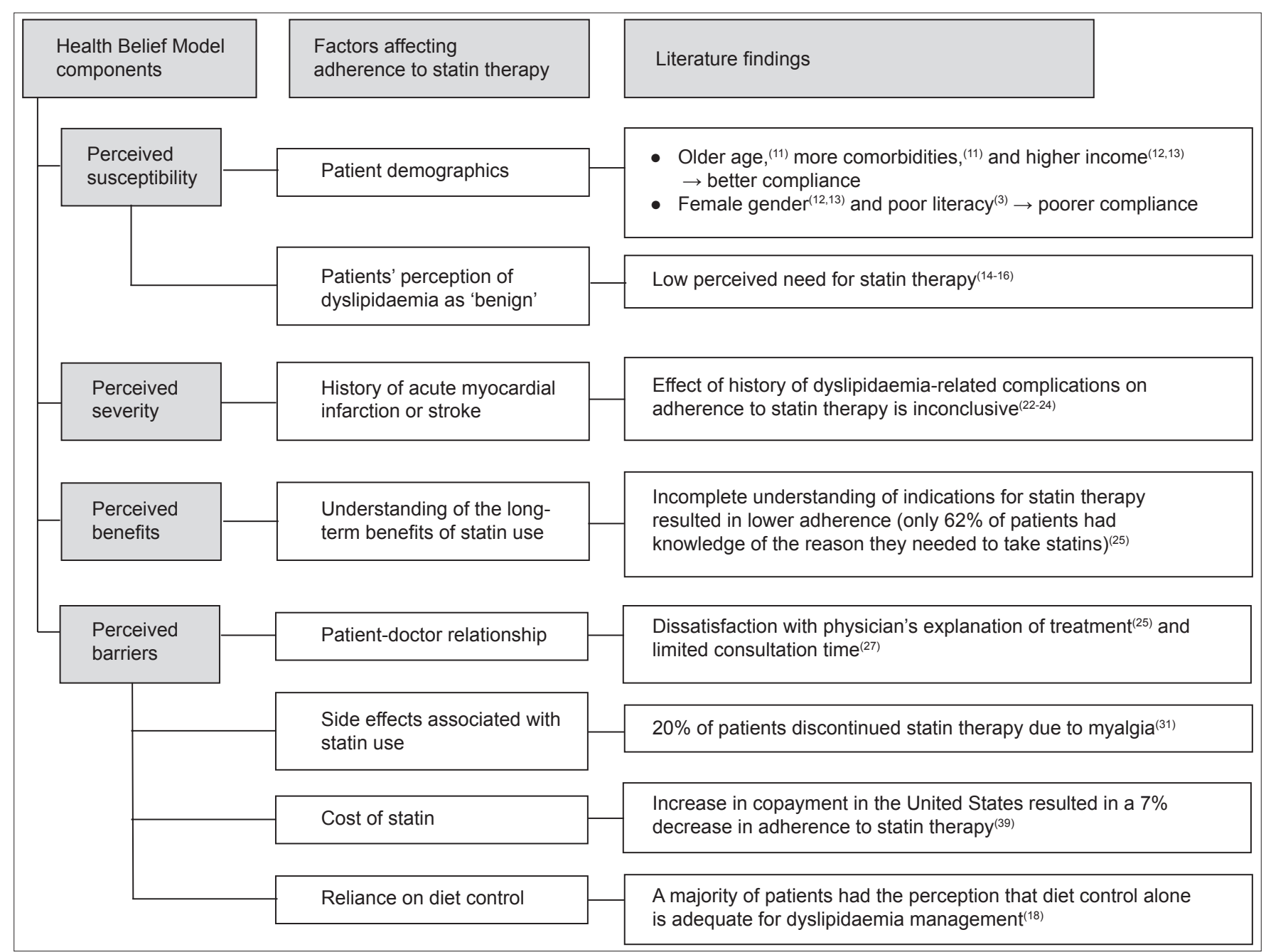

Fig. 1 Flowchart illustrates the use of the Health Belief Model to understand the factors affecting patients' perception on the use of statins in the management of dyslipidaemia.

therapy among a comparative group of patients without acute myocardial infarction or stroke, nor did they explore the patients' perceived severity of and susceptibility to dyslipidaemia-related complications after a cardiovascular or cerebrovascular event. As such, our understanding of the true impact a history of adverse events due to dyslipidaemia-related complications has on statin adherence may be limited.

The regularity of statin use in the management of dyslipidaemia also depends on the patient's understanding of the benefits statins confer on the prevention of adverse health outcomes (i.e. the patient's perceived benefits of the treatment). A qualitative study conducted by Fung et $\mathrm{al}^{(16)}$ showed that participants did not fully understand why statins were prescribed and questioned the actual need to consume the medication. Gialamas et al $^{(25)}$ likewise found that only $62 \%$ of patients fully understood why they needed to take cholesterol-lowering medications. This lack of patient understanding regarding the benefits of cholesterol-lowering medications was also highlighted by Lytsy and Westerling, ${ }^{(26)}$ who observed that some patients appeared to have difficulty understanding the preventive effect of statins.

Perceived barriers also play a role in patient adherence to statin therapy. One of the key barriers may be related to the perceived lack of communication between the physician and patient during consultation. ${ }^{(4)}$ A majority of participants in the focus group discussions conducted by Fung et al stated that they did not receive details about statins from their physicians. ${ }^{(16)}$ Similarly, a study by Gialamas et al reported that only $69 \%$ of the study participants were satisfied with the information on cholesterol-lowering medications given by their primary care physicians. ${ }^{(25)}$ Indeed, the amount of information regarding new medications successfully transmitted to patients is inadequate, possibly due to limited consultation time. (27) Patients' lack of understanding and knowledge about lipid-lowering drugs may translate to poor patient satisfaction and consequently, lower medication adherence. ${ }^{(26)}$

Another important barrier to the use of statins is the side effects associated with it. A serious, but rare, side effect is rhabdomyolysis. ${ }^{(28)}$ Another less serious, but more common, side effect is myalgia without a rise in creatine phosphokinase. ${ }^{(28)}$ Ucar et al suggested that myalgia accounts for up to $25 \%$ of all adverse events related to statin use, ${ }^{(29)}$ resulting in reduced patient compliance and increased statin discontinuation. ${ }^{(30)}$ The Prediction of Muscular Risk on Observational Conditions study, ${ }^{(31)}$ which involved 7,394 French patients with dyslipidaemia, showed that $19.8 \%$ of participants discontinued statin therapy and $16.7 \%$ reduced their statin dose due to muscular side effects. 
Patient compliance to medications may also be directly associated with the patient's lack of information on the duration of treatment. Yilmaz et al suggested that informing patients about treatment duration may improve medication adherence. ${ }^{(32)}$ Unfortunately, Mann et al found that discussions regarding the duration of statin therapy occurred in only $17 \%$ of new consultations. ${ }^{(18)}$ This suggests that the number of patients who are aware of the duration of statin therapy is low. ${ }^{(33)}$ Patients' lack of awareness regarding the need for long-term statin therapy for secondary prevention may also potentiate statin nonadherence, especially since dyslipidaemia is an asymptomatic disease.

Patients' preference for diet control over pharmacotherapy is also a potential barrier to compliance to medications. Mann et al showed that nearly $40 \%$ of 71 first-time statin users preferred to use diet control before initiating statin therapy (the baseline cholesterol level of the study participants were not known), and $70 \%$ of these patients thought that diet could completely resolve their dyslipidaemia. ${ }^{(18)}$ This perception could partly be attributed to a general dislike for taking medications, hearsay about the possible unpleasant side effect associated with statin use based on the experience of family and friends, and exposure to undesirable influence of the media (e.g. television and the Internet). ${ }^{(16)}$ The substantial proportion of patients who prefer diet therapy over statin therapy may be a cause for concern, depending on the individual's baseline cholesterol level at diagnosis. Despite the strong recommendation for diet as a treatment for dyslipidaemia, ${ }^{(34)}$ a review by Varady and Jones, ${ }^{(35)}$ which looked at the efficacy of diet and exercise in treating dyslipidaemia, showed that while the combination of a low-fat diet and regular exercise resulted in lower total cholesterol and LDL levels, such treatment should only be implemented in place of pharmacotherapy when cholesterol levels are slightly elevated. If a patient's serum cholesterol is greater than $6.5 \mathrm{mmol} / \mathrm{L}$, diet control alone is unlikely to be effective. ${ }^{(36)}$ Furthermore, a randomised controlled trial by Jenkins et $\mathrm{al}^{(37)}$ revealed that diet control reduced LDL levels by only $8.0 \%$, whereas lovastatin reduced LDL levels by $30.9 \%$. Depending on the patient's baseline cholesterol level, initiating diet therapy alone may not be optimum for the management of dyslipidaemia.

The cost of statins has always been a critical barrier in the maintenance of adherence. In a meta-analysis of 132 studies that examined the relationship between the cost of medications and adherence, an increase in cost was associated with poor adherence and a higher likelihood of discontinuation of therapy. ${ }^{(38)}$ In another study performed in the United States, a rise in copayment for lipid-lowering medication from US\$2 to US\$7 resulted in a $7 \%$ reduction in compliance rates. ${ }^{(39)}$

Several interventions targeting improvement in medication adherence have been studied. Less than half of these interventions actually led to a consistent increase in adherence, ${ }^{(40,41)}$ implying that there is no single strategy that can address all the factors that influence patients' perceptions on the use of statins to treat dyslipidaemia. Multimodal interventions, however, have shown great potential in improving adherence and outcomes. ${ }^{(42)}$ In a randomised controlled trial by Lee et $\mathrm{al}^{\left({ }^{(43)}\right.}$ the authors found that patient education by pharmacists, medication reminder packages and regular follow-up resulted in an approximately $30 \%$ improvement in medication adherence, systolic blood pressure and LDL cholesterol level.

\section{DISCUSSION}

Although there is extensive interest in identifying the factors affecting adherence to statin therapy, there are relatively few studies that specifically evaluate patients' perceptions toward statin use. Among these studies, even fewer incorporate behavioural models as a means of consolidating factors affecting patients' perceptions. In the present literature review, apart from evaluating adherence to statin therapy using the patients' demographic characteristics, we also assessed the factors influencing patients' perception of statin therapy using the HBM. In view of the multitude of factors, we propose a patient-centric approach that aims to raise patients' perceived susceptibility to dyslipidaemia-related complications, correct misconceptions that may potentially reduce statin adherence, and improve physician-patient relationships.

The first component of our proposed approach involves assessing the patients' perceived susceptibility to dyslipidaemiarelated complications early in the course of treatment. A relevant tool that may be applied is the Illness Perception Score, ${ }^{(44)}$ which identifies the patients' perception of the symptoms associated with the disease, the aetiology of disease, the expected duration of illness, as well as the anticipated outcomes of treatment. This tool has been used in the management of several chronic diseases, including rheumatoid arthritis, ${ }^{\left({ }^{45)}\right.}$ psoriasis, ${ }^{\left({ }^{46)}\right.}$ chronic obstructive pulmonary disease ${ }^{(47)}$ and diabetes mellitus. ${ }^{(48)}$ Evaluation of patients' views using this scoring assessment showed that the longer the expected duration of treatment, the more severe the perception regarding the risks of disease complication. ${ }^{(49)}$ Using expected treatment duration as a surrogate, this tool can potentially identify patients who may have lower perceived susceptibility to dyslipidaemia-related complications. On identification of such patients, physicians can then intensify their efforts to inculcate an awareness of the patients' susceptibility to complications, as well as promote the need for long-term adherence to medications among these patients.

To further enhance patients' perceived susceptibility to cardiovascular risks and dyslipidaemia-related complications, education tools that employ engaging visual aids depicting complications may be utilised. For instance, Goldman et al studied patients' responses to an innovative strategy called HeartAge, which involves showing patients a simple bar graph demonstrating that the 10 -year probability of an acute cardiovascular event of a 42-year-old man with cardiovascular risk factors was equivalent to that of a 70-year-old man based on the Framingham Heart Study. ${ }^{(15)}$ In a subsequent focus group discussion, participants in Goldman et al's study supported this education strategy, stating that it provided them with the motivation to change their behaviours. ${ }^{(15)}$

As highlighted in the Results of this article, the impact of perceived severity of dyslipidaemia on adherence to statin therapy remains inconclusive. To address this, physicians should 
be encouraged to explore the perceived severity of disease among patients who have suffered from dyslipidaemia-related complications and compare their perception against that of asymptomatic patients with dyslipidaemia. The rates of adherence to statin therapy of the two patient groups are then closely monitored via documentation of their frequency of statin refills. Establishing the impact of perceived severity of dyslipidaemia on adherence to statin therapy is important, as it will assist physicians in customising lipid-lowering therapy for individual patients. Furthermore, we believe that patients who have experienced dyslipidaemia-related complications, such as acute myocardial infarction, may require even more intensive education regarding the benefits of statins for preventing further complications.

The present literature review also reveals a gap between patients' perception of diet control and the actual efficacy of diet control alone in the management of dyslipidaemia. While physicians may acknowledge patients' preference for diet control, it is of paramount importance to correct any misconceptions that patients may have about diet vs. statin therapies, and to convince such patients, particularly high-risk patients, of the need to initiate pharmacotherapy when appropriate. As part of a multipronged approach targeted at correcting dietary misconceptions in the management of dyslipidaemia, patient-friendly visual aids, such as charts and bar graphs, may be used to illustrate the efficacy of diet control alone as opposed to statin therapy in lowering LDL cholesterol. After being shown these visual aids, patients should then be encouraged to take their prescribed statins and return for a follow-up lipid profile. This will allow patients to make a more concrete comparison between diet control alone and statin therapy.

The perceived lack of communication between patients and their physician is a key barrier that may affect the optimal management of dyslipidaemia. Zolnierek and Dimatteo found that if a physician communicates effectively, the likelihood of patient adherence is 2.16 times higher. ${ }^{(50)}$ Lengthening consultation time may be one way to improve physicianpatient communication. However, this may not be feasible or applicable for all categories of patients in view of the limited healthcare resources in many countries. Patient-risk stratification conducted prior to consultation may allow physicians to spend more time communicating with patients who have a greater risk for nonadherence. A multidisciplinary approach involving nurse educators and other allied healthcare professionals, such as pharmacists, will provide patients with additional sources of information to approach for clarification regarding their illness and treatment. Medical colleges with robust training programmes that equip physicians with better communication skills will form part of the complex interventions. ${ }^{(51)}$

While the cost of statins has been shown to be a potential barrier, it is important to recognise that patients' willingness to pay for medications may also be influenced by their perceived severity of dyslipidaemia and level of understanding of the preventive role of statins. A patient with inadequate knowledge regarding the efficacy of statins in reducing dyslipidaemia-related complications may be less motivated to be compliant with statin therapy, and thus less willing to pay for the medication. This highlights the importance of patient education, where cost-benefit analyses may be applied to address patients' misconceptions regarding the cost of statins while providing opportunities for physicians to reinforce the importance of compliance to statin therapy. In addition to patient education, we recommend routine screening of patients' financial statuses, as this can help medical social workers identify needy patients who require financial intervention.

The effectiveness of our proposed patient-centric approach on the rate of adherence to statin therapy awaits further evaluation. In view of the significant public health impact that can be derived from better adherence to statin therapy, our future direction includes prioritising efforts to conduct studies using our proposed patient-centric approach as a framework and constantly evaluating its effectiveness in producing desirable clinical outcomes in dyslipidaemia management.

To conclude, future multifaceted interventions addressing the lack of adherence to statin therapy need to consider patients' perceived severity of and susceptibility to dyslipidaemia-related complications, as well as their perceived benefits of and barriers to the use of statins. Physicians and healthcare professionals should make a concerted effort to optimise patients' understanding of the efficacy and benefits of statin therapy, clarify misconceptions regarding statin therapy, and remove barriers to the use of statins. This can be achieved through focused education initiatives, fostering of stronger healthcare partnerships and shared decisionmaking between physicians and patients.

\section{REFERENCES}

1. Baigent C, Keech A, Kearney PM, et al. Efficacy and safety of cholesterollowering treatment: prospective meta-analysis of data from 90,056 participants in 14 randomised trials of statins. Lancet 2005; 366:1267-78.

2. Cholesterol Treatment Trialists' (CTT) Collaborators, Mihaylova B, Emberson J, et al. The effects of lowering LDL cholesterol with statin therapy in people at low risk of vascular disease: meta-analysis of individual data from 27 randomised trials. Lancet 2012; 380:581-90.

3. Bates TR, Connaughton VM, Watts GF. Non-adherence to statin therapy: a major challenge for preventive cardiology. Expert Opin Pharmacother 2009; 10:2973-85

4. Ho KT, Chin KW, Ng KS, et al. The A-SACT (Achievement in Singapore of Cholesterol Targets) study in patients with coronary heart disease. Am J Cardiovasc Drugs 2006; 6:383-91.

5. Ansell BJ. Not getting to goal: the clinical costs of noncompliance. J Manag Care Pharm 2008; 14:9-15.

6. Sokol MC, McGuigan KA, Verbrugge RR, Epstein RS. Impact of medication adherence on hospitalization risk and healthcare cost. Med Care 2005; 43:521-30.

7. Chaudhry HJ, McDermott B. Recognizing and improving patient nonadherence to statin therapy. Curr Atheroscler Rep 2008; 10:19-24.

8. Ford I, Murray H, Packard CJ, et al. Long-term follow-up of the West of Scotland Coronary Prevention Study. N Engl J Med 2007; 357:1477-86.

9. Benner JS, Glynn RJ, Mogun H, et al. Long-term persistence in use of statin therapy in elderly patients. JAMA 2002; 288:455-61.

10. Rosenstock IM, Strecher VJ, Becker MH. Social learning theory and the Health Belief Model. Health Educ Q 1988; 15:175-83.

11. Wong MC, Jiang JY, Griffiths SM. Adherence to lipid-lowering agents among 11,042 patients in clinical practice. Int J Clin Pract 2011; 65:741-8.

12. Mann DM, Woodward M, Muntner P, Falzon L, Kronish I. Predictors of nonadherence to statins: a systematic review and meta-analysis. Ann Pharmacother 2010; 44:1410-21.

13. Chan DC, Shrank WH, Cutler D, et al. Patient, physician, and payment predictors of statin adherence. Med Care 2010; 48:196-202.

14. Durack-Bown I, Giral P, d'Ivernois JF, et al. Patients' and physicians' 
perceptions and experience of hypercholesterolaemia: a qualitative study. Br J Gen Pract 2003; 53:851-7.

15. Goldman RE, Parker DR, Eaton CB, et al. Patients' perceptions of cholesterol, cardiovascular disease risk, and risk communication strategies. Ann Fam Med 2006; 4:205-12.

16. Fung $\mathrm{V}$, Sinclair $\mathrm{F}$, Wang $\mathrm{H}$, et al. Patients' perspectives on nonadherence to statin therapy: a focus-group study. Perm J 2010; 14:4-10.

17. McHorney CA. The Adherence Estimator: a brief, proximal screener for patient propensity to adhere to prescription medications for chronic disease. Curr Med Res Opin 2009; 25:215-38.

18. Mann DM, Allegrante JP, Natarajan S, Halm EA, Charlson M. Predictors of adherence to statins for primary prevention. Cardiovasc Drugs Ther 2007; 21:311-6.

19. McClendon D. Perceived Susceptibility of Cardiovascular Disease as a Moderator of Relationships between Perceived Severity and Cardiovascular Health Promoting Behaviors among Female Registered Nurses (dissertation). Atlanta, GA: Georgia State University, 2011.

20. DiMatteo MR, Haskard KB, Williams SL. Health beliefs, disease severity, and patient adherence: a meta-analysis. Med Care 2007; 45:521-8.

21. Yan J, You LM, He JG, et al. Illness perception among Chinese patients with acute myocardial infarction. Patient Educ Couns 2011; 85:398-405

22. Choudhry NK, Setoguchi S, Levin R, Winkelmayer WC, Shrank WH. Trends in adherence to secondary prevention medications in elderly post-myocardial infarction patients. Pharmacoepidemiol Drug Saf 2008; 17:1189-96.

23. Shah ND, Dunlay SM, Ting $\mathrm{HH}$, et al. Long-term medication adherence after myocardial infarction: experience of a community. Am J Med 2009; 122:961.e7-13.

24. Colivicchi F, Bassi A, Santini M, Caltagirone C. Discontinuation of statin therapy and clinical outcome after ischemic stroke. Stroke 2007; 38:2652-7

25. Gialamas A, Aylward P, Vanlint S, Stocks NP. Cholesterol lowering medication - patients' knowledge, attitudes and experiences. Aust Fam Physician 2011; 40:519-22

26. Tarn DM, Paterniti DA, Kravitz RL, et al. How much time does it take to prescribe a new medication? Patient Educ Couns 2008; 72:311-9.

27. Lytsy $P$, Westerling R. Patient expectations on lipid-lowering drugs. Patient Educ Couns 2007; 67:143-50.

28. McKenney JM, Davidson MH, Jacobson TA, Guyton JR; National Lipid Association Statin Safety Assessment Task Force. Final conclusion and recommendations of the National Lipid Association Statin Safety Assessment Task Force. Am J Cardiol 2006; 97:89C-94C.

29. Ucar M, Mjörndal T, Dahlqvist R. HMG-CoA reductase inhibitors and myotoxicity. Drug Saf 2000; 22:441-57.

30. Chapman MJ, Carrie A. Mechanisms of statin-induced myopathy: a role for the ubiquitin-proteasome pathway? Arterioscler Thromb Vasc Biol 2005; 25:2441-4

31. Bruckert E, Hayem G, Dejager S, Yau C, Bégaud B. Mild to moderate muscular symptoms with high-dosage statin therapy in hyperlipidemic patients--the PRIMO study. Cardiovasc Drugs Ther 2005; 19:403-14.

32. Yilmaz MB, Pinar $M$, Naharci I, et al. Being well-informed about statin is associated with continuous adherence and reaching targets. Cardiovasc Drugs Ther 2005; 19:437-40.
33. Yilmaz MB, Biyikoglu SF, Guray Y, et al. Level of awareness of on-treatment patients about prescribed statins. Cardiovasc Drugs Ther 2004; 18:399-404.

34. National Heart, Lung, and Blood Institute. Third report of the National Cholesterol Education Program (NCEP) Expert Panel on Detection, Evaluation, and Treatment of High Blood Cholesterol in Adults (Adult Treatment Panel III); 2001. NIH Publication No. 01-3670.

35. Varady KA, Jones PJ. Combination diet and exercise interventions for the treatment of dyslipidemia: an effective preliminary strategy to lower cholesterol levels? J Nutr 2005; 135:1829-35.

36. Ramsay LE, Yeo WW, Jackson PR. Dietary reduction of serum cholesterol concentration: time to think again. BMJ 1991; 303:953-7.

37. Jenkins DJ, Kendall CW, Marchie A, et al. Effects of a dietary portfolio of cholesterol-lowering foods vs lovastatin on serum lipids and C-reactive protein. JAMA 2003; 290:502-10.

38. Goldman DP, Joyce GF, Zheng Y. Prescription drug cost sharing: associations with medication and medical utilization and spending and health. JAMA 2007; 298:61-9.

39. Doshi JA, Zhu J, Lee BY, Kimmel SE, Volpp KG. Impact of a prescription copayment increase on lipid-lowering medication adherence in veterans. Circulation 2009; 119:390-7.

40. Kripalani S, Yao X, Haynes RB. Interventions to enhance medication adherence in chronic medical conditions: a systematic review. Arch Intern Med 2007; 167:540-50

41. Choudhry NK, Winkelmayer WC. Medication adherence after myocardial infarction: a long way left to go. J Gen Intern Med 2008; 23:216-8.

42. Ho PM, Bryson CL, Rumsfeld JS. Medication adherence: its importance in cardiovascular outcomes. Circulation 2009; 119:3028-35.

43. Lee JK, Grace KA, Taylor AJ. Effect of a pharmacy care program on medication adherence and persistence, blood pressure, and low-density lipoprotein cholesterol: a randomized controlled trial. JAMA 2006; 296:2563-71.

44. Weinman J, Petrie KJ, Moss-Morris R, Horne R. The illness perception questionnaire: a new method for assessing the cognitive representation of illness. Psychol Health 1996; 11:114-29.

45. Scharloo M, Kaptein AA, Weinman JA, et al. Predicting functional status in patients with rheumatoid arthritis. J Rheumatol 1999; 26:1686-93.

46. Scharloo M, Kaptein AA, Weinman J, et al. Patients' illness perceptions and coping as predictors of functional status in psoriasis: a 1-year follow-up. Br J Dermatol 2000; 142:899-907.

47. Scharloo M, Kaptein AA, Weinman JA, Willems LN, Rooijmans HG. Physical and psychological correlates of functioning in patients with chronic obstructive pulmonary disease. J Asthma 2000; 37:17-29.

48. Griva K, Myers LB, Newman S. Illness perceptions and self efficacy beliefs in adolescents and young adults with insulin dependent diabetes mellitus. Psychol Health 2000; 15:733-50.

49. Petrie KJ, Weinman J, Sharpe N, Buckley J. Role of patients' view of their illness in predicting return to work and functioning after myocardial infarction: longitudinal study. BMJ 1996; 312:1191-4.

50. Zolnierek KB, Dimatteo MR. Physician communication and patient adherence to treatment: a meta-analysis. Med Care 2009; 47:826-34.

51. Casebeer L, Huber C, Bennett N, et al. Improving the physician-patient cardiovascular risk dialogue to improve statin adherence. BMC Fam Pract 2009; 10:48. 